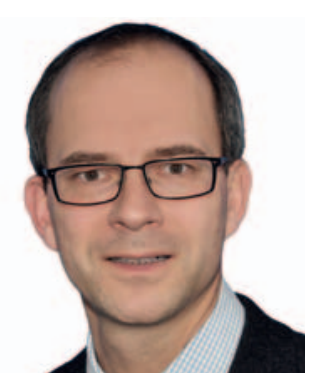

\title{
Chronische spontane Urtikaria bei Asthmatikern: Das Rätsel um reziproke Mechanismen
}

\section{Christian Vogelberg}

Klinik und Poliklinik für Kinder- und Jugendmedizin, Universitäts AllergieCentrum (UAC) Dresden, Dresden

Abstractübersetzung aus Vadasz Z, Kessel A, Hershko AY, Maurer M, Toubi E: Seasonal exacerbation of asthma is frequently associated with recurrent episodes of acute urticaria. Int Arch Allergy Immunol 2016;169:263-266.

\section{Saisonale Exazerbationen von Asthma sind häufig mit wiederkehrenden Episoden akuter Urtikaria assoziiert}

\section{Schlüsselwörter}

Urtikaria · Asthma · Saisonale Allergie

\section{Zusammenfassung}

Hintergrund: Asthma und Urtikaria werden beide zum Teil durch die vermehrte Ausschüttung von Histamin aus hochgradig aktivierten Mastzellen vermittelt. Die Pathophysiologie ist jedoch verschieden, da der Mastzelldegranulation jeweils unterschiedliche Mechanismen zugrunde liegen.

Ziel: Untersuchung der Inzidenz von Urtikaria bei Patienten mit Asthma und der von Asthma bei Patienten mit chronischer spontaner Urtikaria (CSU).

Patienten und Methoden: Über einen Beobachtungszeitraum von einem Jahr wurden bei Patienten mit Asthma $(n=110)$ die
Inzidenz von Urtikaria und gegebenenfalls deren Merkmale sowie ein Zusammenhang mit saisonalen Exazerbationen, falls vorhanden, und der Schwere des Asthmas untersucht. Im selben Zeitraum untersuchten wir prospektiv Patienten mit CSU $(n=95)$ auf die Inzidenz von Asthma. Gesunde Probanden ( $n=100)$, die als Kontrollgruppe dienten, wurden ebenfalls untersucht.

Ergebnisse: Urtikaria-Episoden traten bei 26/110 Asthmapatienten auf (23,6\%), aber bei nur 2/100 gesunden Kontrollpersonen (2\%) ( $p<0,0001$ ). In dem einjährigen Beobachtungszeitraum kamen Urtikaria-Episoden signifikant häufiger bei Asthmapatienten mit positiven Reaktionen im Haut-Prick-Test (hauptsächlich auf saisonale Pollen) vor und häuften sich dementsprechend während saisonaler Asthma-Exazerbationen, d.h. während akuter Episoden von Urtikaria. Die Asthmainzidenz bei CSU-Patienten betrug 10,5\%, ähnlich wie in der gesunden Kontrollgruppe.

Diskussion: Unsere Studie erbringt erstmals den Nachweis, dass Asthmapatienten häufig akute Urtikaria entwickeln, besonders während saisonaler Exazerbationen. CSU-Patienten hingegen zeigen keine erhöhte Inzidenz von Asthma.

(c) 2017 S. Karger GmbH, Freiburg

\section{KARGER}

Fax +497614520714 information@karger.com www.karger.com

\section{(C) 2017 S. Karger GmbH, Freiburg}




\section{Transfer in die Praxis}

\section{Hintergrund}

Die Therapie mit dem Anti-IgE-Antikörper Omalizumab zeigt bei zwei Erkrankungen besondere therapeutische Effektivität - dem schweren allergischen Asthma und der chronisch spontanen Urtikaria (CSU). Wenngleich die beiden Erkrankungen nicht unmittelbar miteinander vergleichbar sind und unterschiedliche pathophysiologische Mechanismen dominieren, so gibt es dennoch einzelne Überschneidungen, die zumindest teilweise einen Erklärungsansatz für die Effektivität der Behandlung liefern könnten. Dies umfasst neben IgE z.B. Mediatoren, die bei beiden Erkrankungen eine Relevanz haben, wie IL4, IL10, IL33 und B-Zell-aktivierender Faktor. Doch ist die Prävalenz von Urtikaria bei Asthmapatienten wirklich erhöht?

\section{Studienergebnisse}

Die vorliegende Studie aus Israel untersuchte über ein Jahr hinweg 110 Asthmatiker und 95 Patienten mit CSU und verglich diese mit 100 Kontrollpatienten. Mit rund 24\% trat Urtikaria bei den Asthmapatienten signifikant häufiger auf als in der Kontrollgruppe. Besonders häufig traten die vergleichsweise kurzen Episoden im Kontext von Asthmaexazerbationen, bei moderatem bis schwerem Asthma und Sensibilisierungen und Exposition gegenüber lokalen Aeroallergenen auf. Die Rate an Asthma bei Patienten mit CSU war jedoch überraschenderweise nicht höher als in der Kontrollgruppe.

\section{Fazit für die Praxis}

Zunächst einmal untermauert die Studie eine interessante Beobachtung, wenngleich die diskutierten Erklärungsansätze keine Antwort darauf geben, warum die Assoziationen nur einseitig vorliegen. Erhöhte Konzentrationen einzelner Substanzen wie z.B. IL33, IgE, ECP oder VEGF (vascular endothelial growth factor) und ihr Effekt auf einzelne Zellen können sicherlich nur Erklärungsansätze liefern. Genauso relevant ist die Frage, warum Mastzellen der Haut offensichtlich anders bei entsprechendem Allergen- und Mediatorkontakt reagieren als Mastzellen der Atemwege. Für die Klärung dieser Fragen sind weitere Untersuchungen nötig, die auch differenziertere und standardisierte Patientengruppen einbeziehen, sowohl was das Sensibilisierungsmuster anbelangt als auch den Asthmaschweregrad. Auch die Therapie und Komorbitäten, insbesondere aus dem atopischen Formenkreis, sollten dabei Berücksichtigung finden.

\section{Disclosure Statement}

Hiermit erkläre ich, dass keine Interessenskonflikte in Bezug auf den vorliegenden Wissenstransfer bestehen.

Kontaktadresse: Prof. Dr. Christian Vogelberg, Klinik und Poliklinik für Kinder- und Jugendmedizin, Universitäts AllergieCentrum (UAC) Dresden, Fetscherstraße 74, Haus 21, 01307 Dresden, Deutschland, christian.vogelberg@uniklinikum-dresden.de 